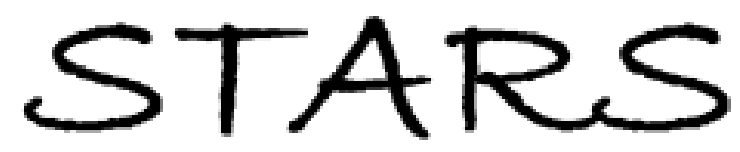

University of Central Florida

STARS

$1-1-2013$

\title{
Low voltage and high contrast blue phase liquid crystal with red- shifted Bragg reflection
}

Jin Yan

University of Central Florida

Zhenyue Luo

Shin-Tson Wu

University of Central Florida

Jyh-Wen Shiu

Yu-Cheng Lai

See next page for additional authors

Find similar works at: https://stars.library.ucf.edu/facultybib2010

University of Central Florida Libraries http://library.ucf.edu

This Article is brought to you for free and open access by the Faculty Bibliography at STARS. It has been accepted for inclusion in Faculty Bibliography 2010 s by an authorized administrator of STARS. For more information, please contactSTARS@ucf.edu.

\section{Recommended Citation}

Yan, Jin; Luo, Zhenyue; Wu, Shin-Tson; Shiu, Jyh-Wen; Lai, Yu-Cheng; Cheng, Kung-Lung; Liu, Shih-Hsien; Hsieh, Pao-Ju; and Tsai, Yuan-Chun, "Low voltage and high contrast blue phase liquid crystal with redshifted Bragg reflection" (2013). Faculty Bibliography 2010s. 4884.

https://stars.library.ucf.edu/facultybib2010/4884

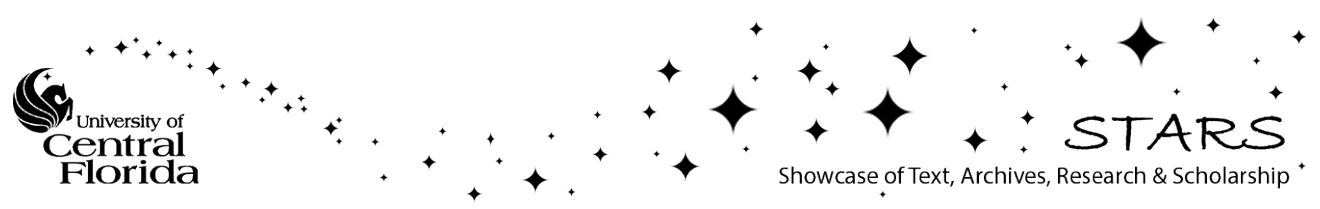




\section{Authors}

Jin Yan, Zhenyue Luo, Shin-Tson Wu, Jyh-Wen Shiu, Yu-Cheng Lai, Kung-Lung Cheng, Shih-Hsien Liu, PaoJu Hsieh, and Yuan-Chun Tsai 


\section{Low voltage and high contrast blue phase liquid crystal with red-shifted Bragg reflection}

Cite as: Appl. Phys. Lett. 102, 011113 (2013); https://doi.org/10.1063/1.4773985

Submitted: 12 November 2012 . Accepted: 17 December 2012 . Published Online: 07 January 2013

Jin Yan, Zhenyue Luo, Shin-Tson Wu, Jyh-Wen Shiu, Yu-Cheng Lai, Kung-Lung Cheng, Shih-Hsien Liu, Pao-Ju Hsieh, and Yuan-Chun Tsai
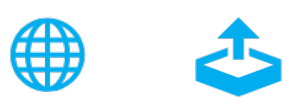

\section{ARTICLES YOU MAY BE INTERESTED IN}

Electro-optics of polymer-stabilized blue phase liquid crystal displays

Applied Physics Letters 94, 101104 (2009); https://doi.org/10.1063/1.3097355

Extended Kerr effect of polymer-stabilized blue-phase liquid crystals

Applied Physics Letters 96, 071105 (2010); https://doi.org/10.1063/1.3318288

Low voltage and high transmittance blue-phase liquid crystal displays with corrugated electrodes

Applied Physics Letters 96, 011102 (2010); https://doi.org/10.1063/1.3290253

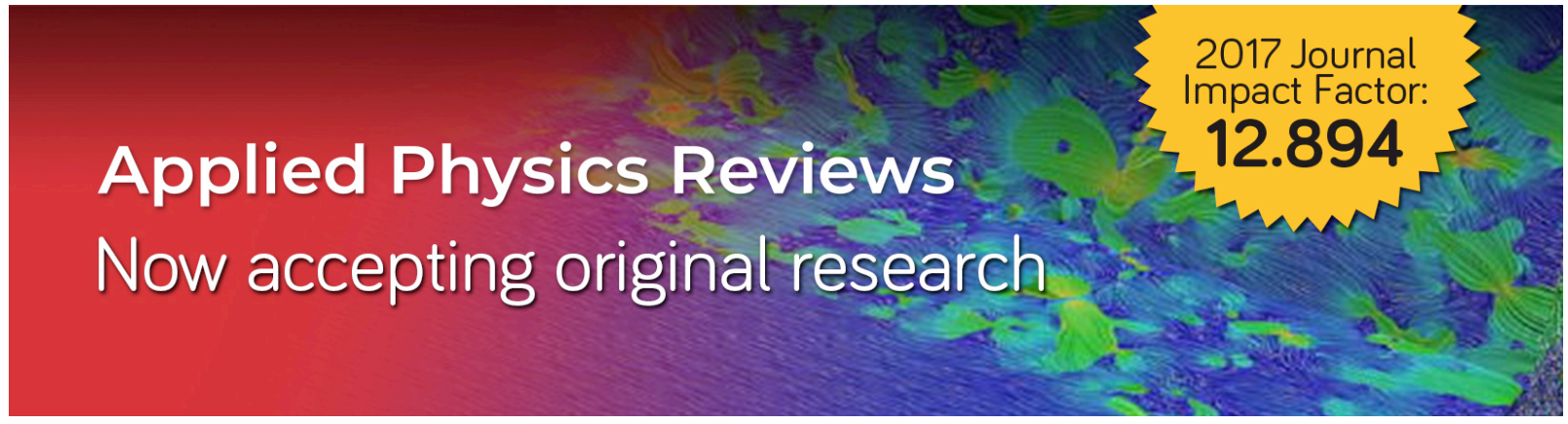




\title{
Low voltage and high contrast blue phase liquid crystal with red-shifted Bragg reflection
}

\author{
Jin Yan, ${ }^{1}$ Zhenyue Luo, ${ }^{1}$ Shin-Tson Wu, ${ }^{1, a)}$ Jyh-Wen Shiu, ${ }^{2}$ Yu-Cheng Lai, ${ }^{2}$ \\ Kung-Lung Cheng, ${ }^{3}$ Shih-Hsien Liu, ${ }^{3}$ Pao-Ju Hsieh, ${ }^{3}$ and Yuan-Chun Tsai ${ }^{3}$ \\ ${ }^{1}$ CREOL, The College of Optics and Photonics, University of Central Florida, Orlando, Florida 32816, USA \\ ${ }^{2}$ Display Technology Center, Industrial Technology Research Institute, Hsinchu, Taiwan \\ ${ }^{3}$ Material and Chemical Research Laboratories, Industrial Technology Research Institute, Hsinchu, Taiwan
}

(Received 12 November 2012; accepted 17 December 2012; published online 7 January 2013)

\begin{abstract}
We demonstrated a low voltage and high contrast polymer-stabilized blue phase liquid crystal (BPLC) by shifting the Bragg reflection to a red wavelength. To retain high contrast ratio, a lefthanded BPLC is sandwiched between a right-handed circular polarizer and a left-handed circular analyzer. The driving voltage is reduced by $35 \%$ as compared to that of a transparent BPLC, while keeping submillisecond response time. The proposed approach would accelerate the emergence of BPLC for next-generation display and photonic applications. (C) 2013 American Institute of Physics. [http://dx.doi.org/10.1063/1.4773985]
\end{abstract}

Polymer-stabilized blue phase liquid crystal (PSBPLC $)^{1-3}$ exhibits several attractive features, such as submillisecond response time, ${ }^{4,5}$ no need for molecular alignment layer, and cell gap insensitivity ${ }^{6,7}$ when in-plane-switching (IPS) electrodes are employed. It is a strong contender for next-generation display and photonic applications. However, the bottleneck is the relatively high operation voltage $(\sim 50 \mathrm{~V})$, which originates from a relatively small Kerr constant of the employed BPLC materials. To reduce the operation voltage to below $10 \mathrm{~V}$, several device structures, such as protruded electrodes, ${ }^{8}$ wall electrodes, ${ }^{9}$ corrugated electrodes, ${ }^{10}$ and vertical field switching mode, ${ }^{11}$ have been proposed. Although these device structures are helpful for reducing voltage, they do increase the manufacturing complexity. Therefore, there is an urgent need to reduce the operation voltage from material composition aspect.

A polymer-stabilized BPLC composite contains three major ingredients: nematic host as switching molecules, chiral agent with a strong helical twisting power to induce blue phases, and mono- and di-functional monomers to stabilize the blue-phase networks. ${ }^{12}$ The Kerr constant of a BPLC composite is primarily governed by three parameters: birefringence $(\Delta \mathrm{n})$, dielectric anisotropy $(\Delta \varepsilon)$, and pitch length $(P)$. Increasing birefringence is a straightforward way to enhance Kerr constant. However, there is a limit because we also have to consider other properties: high resistivity, good UV stability, low melting temperature, and wide nematic range. To satisfy all the above mentioned properties, the $\Delta \mathrm{n}$ is in the vicinity of $\sim 0.2$. Another method to boost Kerr constant is to increase dielectric anisotropy. Indeed, some BPLC hosts with $\Delta \varepsilon>100$ have been recently developed. ${ }^{13,14}$ However, these high $\Delta \varepsilon$ LC hosts usually exhibit a large viscosity which slows down the response time dramatically. Moreover, a high $\Delta \varepsilon$ LC host also causes insufficient charging capability of the active matrix driving circuits, which in turn lowers the transmittance significantly. The third approach is to increase the pitch length. This approach seems simple, but increasing pitch length would shift the Bragg reflection to-

${ }^{a)}$ Electronic mail: swu@ucf.edu. ward the visible spectral region, which in turn degrades the dark state dramatically and leads to a poor contrast ratio. Besides abovementioned factors, the Kerr constant of a BPLC composite can also be improved by optimizing the monomer composition ${ }^{15,16}$ and preparation conditions, such as curing temperature, ${ }^{17} \mathrm{UV}$ intensity, and curing time.

In this paper, we demonstrate a method to enhance the Kerr constant of BPLC by shifting the Bragg reflection from $\sim 350 \mathrm{~nm}$ to $\sim 600 \mathrm{~nm}$ without sacrificing contrast ratio. To retain high contrast ratio, in the experiment we take following precautions: (1) we choose a right-handed circular polarizer (RCP) as the front polarizer so that the transmitted light before entering the BPLC cell is right handed; (2) we use a left-handed chiral dopant so that in the voltage-off state the BPLC cell transmits the incoming right-handed circular light; and (3) we choose a left-handed circular polarizer (LCP) as the analyzer so that the outgoing right-handed circular light from the BPLC cell is absorbed. As a result, we reduce the driving voltage by $35 \%$ while keeping a high contrast ratio and submillisecond response time.

The electro-optical properties of PS-BPLC can be described by extended Kerr effect. ${ }^{18}$ A large Kerr constant helps reduce the driving voltage. From Gerber's model, the Kerr constant under low field assumption can be approximated as ${ }^{19}$

$$
K \sim \frac{\Delta n_{\text {ind }}}{\lambda E^{2}} \approx \Delta n \cdot \Delta \varepsilon \frac{\varepsilon_{0} P^{2}}{k \lambda(2 \pi)^{2}},
$$

where $\Delta n_{\text {ind }}$ is the induced birefringence, $\lambda$ is the wavelength, $\Delta n$ is the intrinsic birefringence of LC host, $k$ is the averaged elastic constant which is affected by both LC host and polymer network, and $P$ is the pitch length. From Eq. (1), the Kerr constant is proportional to $P^{2}$, so increasing the pitch length can effectively improve the Kerr constant. On the other hand, the on-state driving voltage is related to the Kerr constant as ${ }^{13}$

$$
V_{o n}=A \cdot \frac{1}{\sqrt{K}},
$$


where $A$ is the device parameter. Combining Eqs. (1) and (2), and assuming all the other parameters except pitch length remain unchanged, we obtain

$$
V_{\text {on }} \sim \frac{1}{P}
$$

It is desirable to shift the reflection band to near infrared region so that the operating voltage can be reduced significantly and the Bragg reflection does not occur in the visible region. However, one important factor has to be taken into account, which is blue phase appears only when the chirality exceeds a certain value. If the chiral concentration is too low, then the blue phase may not exist. Since the pitch length is inversely proportional to chiral concentration, the pitch length cannot be increased unlimitedly. Moreover, response time will also become slower as pitch length increases. Here, due to the limitation of employed materials, we choose to shift the Bragg reflection wavelength to $\sim 600 \mathrm{~nm}$.

The Bragg reflection wavelength for a PS-BPLC can be expressed as $^{20}$

$$
\lambda=\frac{2 n a}{\sqrt{h^{2}+k^{2}+l^{2}}},
$$

where $n$ and $a$ denote average refractive index and lattice constant of blue phases, and $h, k$, and $l$ are the Miller indices of a crystal plane. For BP-I, the lattice constant $a=P$ and the crystal plane corresponding to the longest reflection peak is (110) plane. Therefore, the longest reflection wavelength is $\lambda_{0}=\sqrt{2} \mathrm{nP}$ and $\lambda_{0}=600 \mathrm{~nm}$ corresponds to a pitch length of $\sim 267 \mathrm{~nm}$ by assuming the average refractive index $n=1.59$.

To compare the performance of short-pitch and longpitch PS-BPLCs, we prepared two samples. The recipes are listed in Table I. The BPLC precursors consist of nematic LC host HTG 135200 (HCCH, China), chiral dopant S5011 ( $\mathrm{HCCH}, \mathrm{HTP} \sim 120 / \mu \mathrm{m}$ ), two kinds of monomers [RM257 (Merck) and TMPTA (1,1,1-trimethylolpropane triacrylate, Sigma Aldrich)], and a small amount of photoinitiator ( $\sim 0.5$ wt. \%, not included in Table I). The nematic LC host has the following properties: $\Delta \mathrm{n}=0.2$ at $\lambda=633 \mathrm{~nm}$, $\Delta \varepsilon=96$ at $1 \mathrm{kHz}$ and $21^{\circ} \mathrm{C}$, and clearing temperature $\mathrm{T}_{\mathrm{c}}=96^{\circ} \mathrm{C}$. The differences between these two samples are the concentrations of chiral dopant and LC host. As a result, the reflection peak of sample I occurs at $\sim 350 \mathrm{~nm}$ and that of sample II at $\sim 600 \mathrm{~nm}$. For each sample, we prepared three IPS cells. The interdigitated electrodes are on the bottom substrate and both the electrode width and gap are $5 \mu \mathrm{m}$. The cell gaps are $\sim 8.5 \mu \mathrm{m}$. The differences between these three cells are threefold: (1) cell 1 has no alignment layer, (2) cell 2 has one rubbed alignment layer on top substrate, and (3) cell 3 has two rubbed alignment layers on both top and bot-

TABLE I. Recipes of two PS-BPLC samples.

\begin{tabular}{lcccc}
\hline \hline Samples & $\begin{array}{c}\text { LC host } \\
\text { (wt. \%) }\end{array}$ & $\begin{array}{c}\text { Chiral dopant } \\
\text { (wt. \%) }\end{array}$ & $\begin{array}{c}\text { RM257 } \\
\text { (wt. \%) }\end{array}$ & $\begin{array}{c}\text { TMPTA } \\
\text { (wt. \%) }\end{array}$ \\
\hline I & 84.52 & 5.48 & 6 & 4 \\
II & 86.85 & 3.15 & 6 & 4 \\
\hline \hline
\end{tabular}

tom substrates. The three cells were prepared under the same conditions. UV curing process was performed near the transition temperature between chiral nematic phase and blue phase with an intensity of $2 \mathrm{~mW} / \mathrm{cm}^{2}$ for $30 \mathrm{~min}$. After UV curing, the clearing temperature of sample I was found to be $\sim 80^{\circ} \mathrm{C}$, while that of sample II was $\sim 90^{\circ} \mathrm{C}$. This difference is because sample II contains less chiral dopant, so its clearing temperature does not drop too much. A wider temperature range is always more preferable.

Figure 1 shows the polarizing optical microscope (POM) images with two crossed linear polarizers. Since the reflection wavelength of sample I is in UV region, it is optically isotropic in the visible region. Therefore, all of the three cells appear dark under crossed polarizers. Here, we choose one cell to represent sample I, as shown in Fig. 1(a). Sample II reflects visible light and the reflection changes the incident polarization state, so that a color image can be observed. For cell 1 without alignment, the blue phase lattices have different orientations (multi-domain) and therefore reflect different colors. As a result, we can observe several colors in the transmission mode [Fig. 1(b)]. The rubbed alignment layers in cell 2 and cell 3 help form a mono-domain texture ${ }^{21,22}$ so that a single color can be observed in Figs. 1(c) and 1(d). From Fig. 1, we found that the dark state of sample II is degraded significantly as compared to sample I.

To obtain a good dark state, we used two broadband and wide-view circular polarizers, as Fig. 2(a) depicts. A white light source was employed and a few collimating lens were used (not shown in the figure). In the experiment, we chose a $\mathrm{RCP}$ as the front polarizer so that the transmitted light was right handed. Next, we used a left-handed chiral dopant so that our PS-BPLC transmitted the incoming right-handed circular light ${ }^{23-26}$ in the voltage-off state. Finally, this righthanded circular light was absorbed by the rear LCP resulting in a good dark state.

Figure 2(b) shows the measured dark state of the six PSBPLC cells between two crossed linear polarizers. Sample I (short-pitch) shows a good dark state, while sample II (long
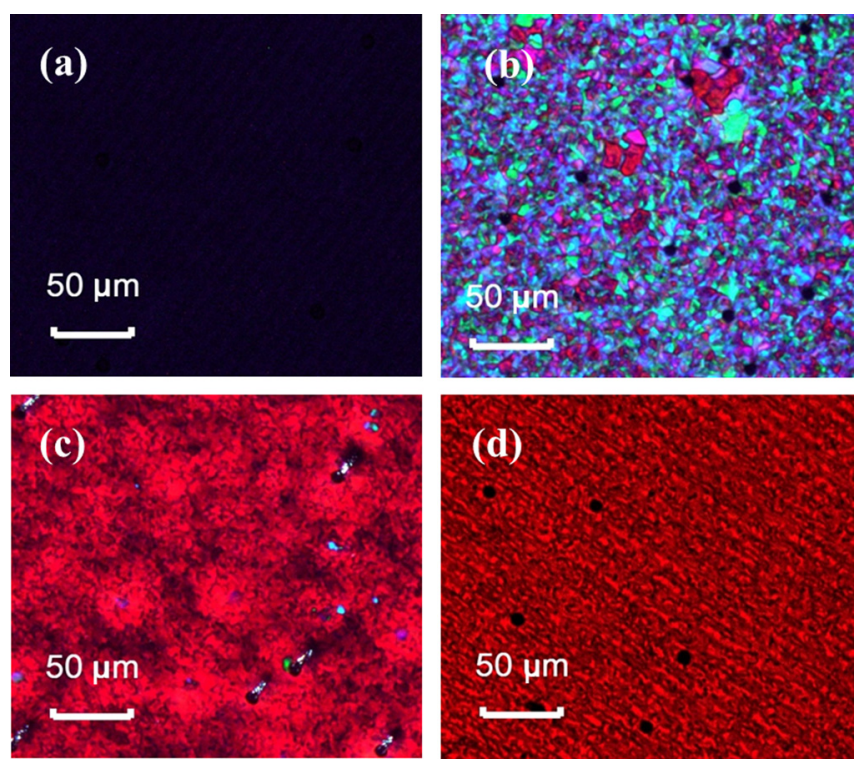

FIG. 1. POM images with two crossed linear polarizers: (a) sample I cell 2, (b) sample II cell 1, (c) sample II cell 2, and (d) sample II cell 3. 

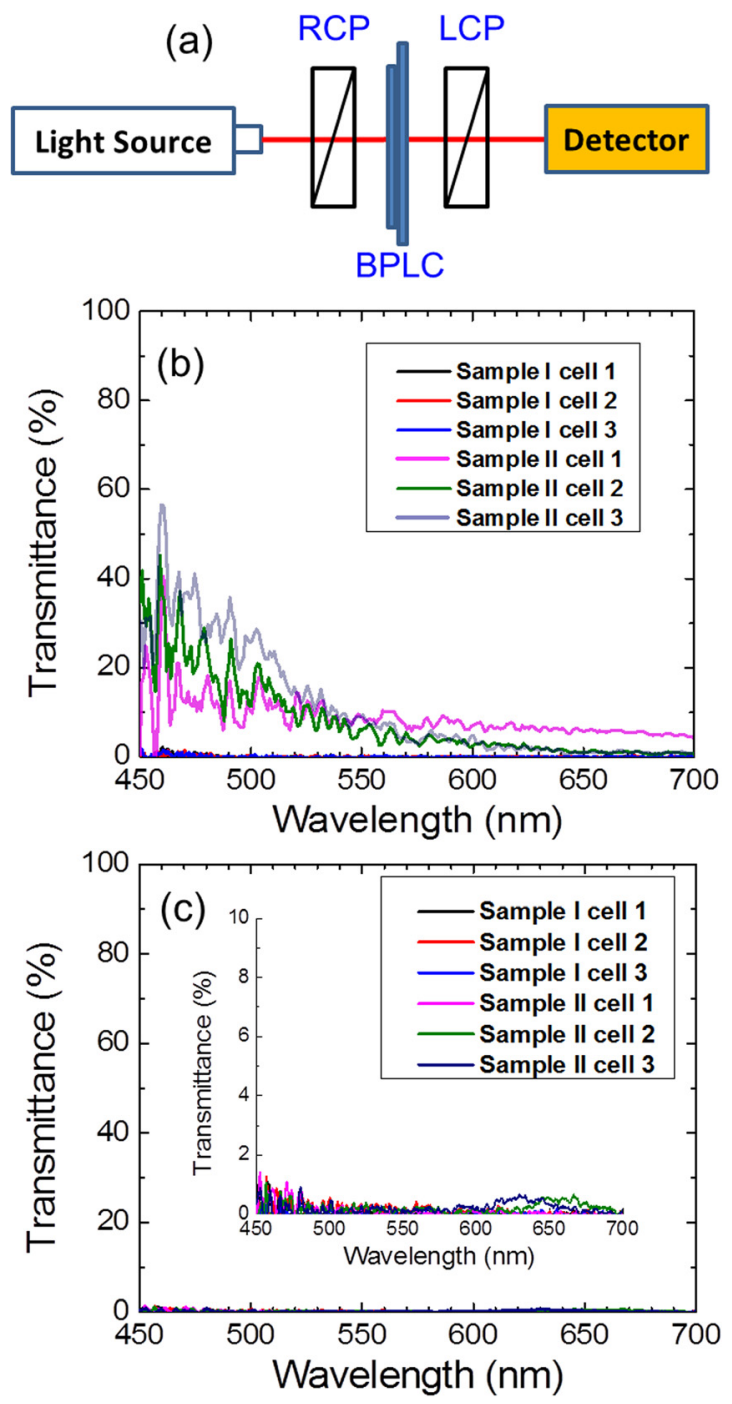

FIG. 2. (a) Diagram of the experimental setup using circular polarizers. RCP: right-handed circular polarizer; LCP: left-handed circular polarizer. (b) Dark state spectrum using two crossed linear polarizers. (c) Dark state spectrum using two crossed circular polarizers.

pitch) exhibits a large light leakage in the visible spectrum. With circular polarizers, the dark state of sample II is greatly improved, as shown in Fig. 2(c).

Figure 3 shows the measured transmittance of four PSBPLC cells in the voltage-off state with a white light source. In Fig. 3(a), a linear polarizer was placed in front of the cells. Sample I is transparent in the displayed spectrum. For sample II, the cell without alignment layer (cell 1) exhibits a fairly large scattering, especially in the shorter wavelength region. Cell 2 and cell 3 show a reflection band near $600 \mathrm{~nm}$. The differences between cell 2 and cell 3 originate from the nonuniformity of the cells due to the capillary filling. In Fig. 3(b), a RCP was placed in front of the cells. With this circular polarizer, the transmittance of sample II is similar to that of sample I. The reason is that these BPLC cells are designed to reflect left-handed circular light so that the right-handed circular light can pass through the cells with high transmittance.

Figure 4 depicts the measured voltage-dependent transmittance (VT) curves using two circular polarizers. An argon-ion laser with $\lambda=514 \mathrm{~nm}$ was employed as probing light source. In the voltage-on state, the BPLC cell behaves
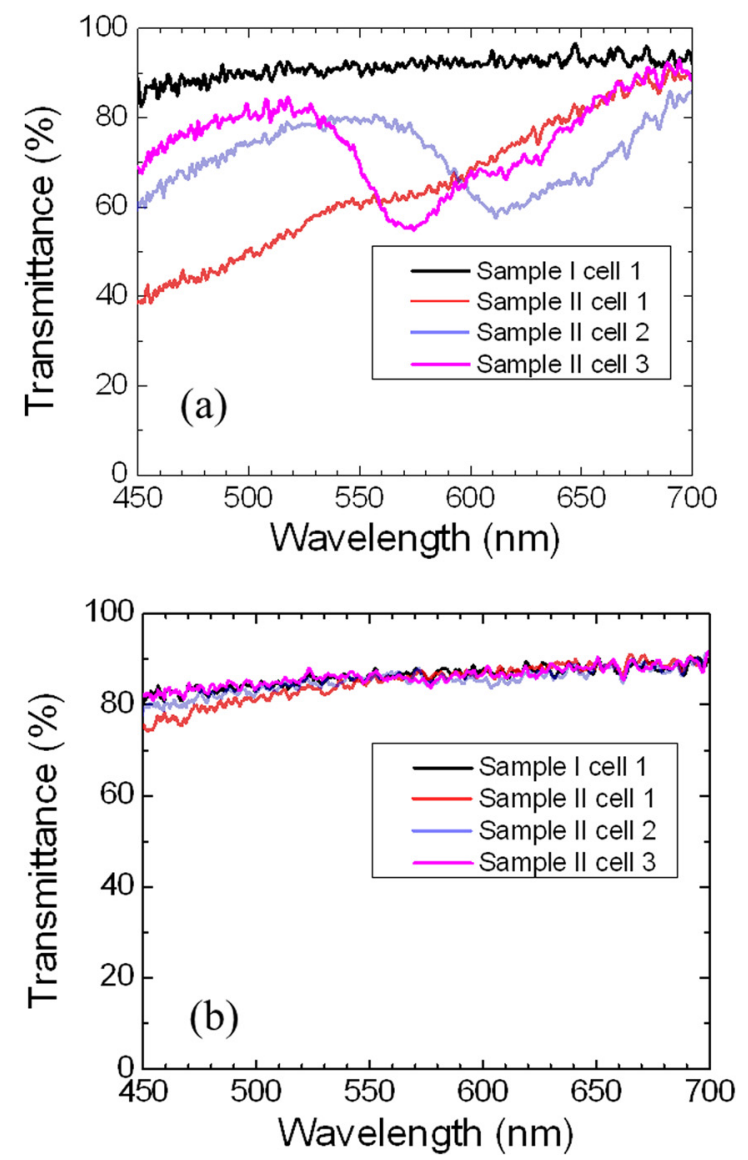

FIG. 3. Measured transmittance of four BPLC cells in the voltage-off state. (a) A linear polarizer is placed in front of the cells. (b) A right-handed circular polarizer is placed in front of the cells.

like a phase grating ${ }^{27}$ and a portion of the energy is transferred to the diffracted orders. Therefore, we used a similar experimental setup to Fig. 2(a) except placing a lens in front of the detector in order to collect the diffracted beams. The measurements were conducted at room temperature $\left(21^{\circ} \mathrm{C}\right)$ and driving frequency $f=1 \mathrm{kHz}$. For sample I, using linear polarizers or circular polarizers make no difference because it is optically isotropic in the visible region. From Fig. 4, sample I cell 1 has an on-state voltage of $54 \mathrm{~V}$ and the corresponding Kerr constant is $K \sim 7.4 \mathrm{~nm} / \mathrm{V}^{2}$. Sample I cell 3

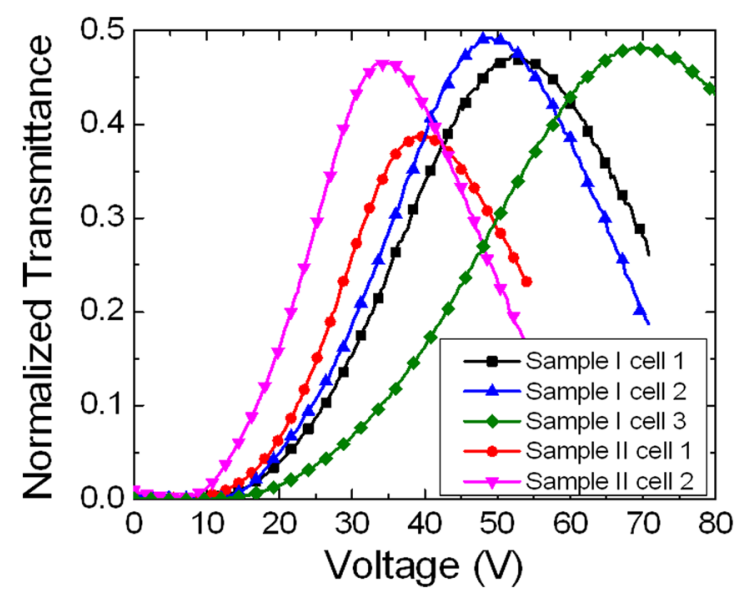

FIG. 4. Measured voltage-dependent transmittance curves using two crossed circular polarizers. $\lambda=514 \mathrm{~nm}, \mathrm{~T}=21^{\circ} \mathrm{C}$, and $f=1 \mathrm{kHz}$. 
requires a much higher voltage than sample I cell 1 , because the alignment layer shields a large portion of the applied voltage, especially for PS-BPLC composites with a large dielectric constant. It is interesting to note that sample I cell 2 has a similar or slightly lower driving voltage than sample I cell 1. This is because the electric fields are concentrated near the bottom substrates so the alignment layer on the top substrate does not shield voltage. Meanwhile, the top alignment layer helps create mono-domain structure to reduce light scattering. Therefore, sample II cell 2 shows a higher transmittance than sample II cell 1 . The uniform lattice orientation also helps to reduce the driving voltage. Most importantly, the driving voltage for sample II cell 2 is only $35 \mathrm{~V}$, which is reduced by $35 \%$ in comparison with sample I cell 1 . The corresponding Kerr constant is $K \sim 17 \mathrm{~nm} / \mathrm{V}^{2}$.

Table II summarizes the performances of the five cells. Transmittance is related to the device structure. The IPS cells we employed have $5-\mu \mathrm{m}$ electrode width and gap. The measured transmittance is slightly lower than the simulated value, which is $\sim 56 \%{ }^{6}$ This is because the lens in front of the detector only collects \pm 1 diffract orders, which carry most of the energy among all the diffracted orders. The energy carried by the higher orders is lost, although it is weak. Higher transmittance can be achieved by modifying device structures or employing an incoherent backlight unit.

Although increasing the pitch length reduces the driving voltage, there are tradeoffs. The first one is that it slows down the response time. The decay time of a BPLC can be approximated as ${ }^{19}$

$$
\tau \approx \frac{\gamma_{1} P^{2}}{k(2 \pi)^{2}}
$$

where $\gamma_{1}$ is the rotational viscosity of the PS-BPLC composite. In Table II, the decay time for sample II is slower than that of sample I due to the longer pitch length, but it is still in the submillisecond range.

The second tradeoff is the larger hysteresis. Hysteresis is related to the peak electric field strength. ${ }^{28,29}$ For a BPLC with an increased pitch length, the lattice structure is looser and easier to be distorted. Therefore, the critical field of sample II should be lower than that of sample I. The hysteresis can be improved by designing smooth electrode shape to reduce the peak electric field. ${ }^{30}$ It is also interesting to notice that cell 2 shows reduced hysteresis than cell 1 for both samples. The strong anchoring from the alignment layer provides a restoring force which might be helpful for reconstructing the BPLC structure during the backward scan. On the other

TABLE II. Summarized performance of five BPLC cells. $\tau$ : decay time; CR: contrast ratio. $\lambda=514 \mathrm{~nm}, \mathrm{~T}=21{ }^{\circ} \mathrm{C}$, and $f=1 \mathrm{kHz}$.

\begin{tabular}{lccccc}
\hline \hline & $\begin{array}{c}\text { Voltage } \\
\left(\mathrm{V}_{\text {rms }}\right)\end{array}$ & $\begin{array}{c}\text { Transmittance } \\
(\%)\end{array}$ & $\begin{array}{c}\tau \\
(\mu \mathrm{s})\end{array}$ & $\begin{array}{c}\text { Hysteresis } \\
(\%)\end{array}$ & $\mathrm{CR}$ \\
\hline Sample I-cell 1 & 54 & 47 & 382 & 5.3 & $6300: 1$ \\
Sample I-cell 2 & 49 & 49 & 324 & 3.5 & $>10000: 1$ \\
Sample I-cell 3 & 70 & 48 & 360 & 5 & $10000: 1$ \\
Sample II—cell 1 & 40 & 39 & 648 & 8 & $300: 1$ \\
Sample II—cell 2 & 35 & 47 & 814 & 6 & $6500: 1$ \\
\hline \hline
\end{tabular}

hand, the larger hysteresis of sample I cell 3 compared to cell 2 might be due to the higher applied voltage.

Contrast ratio is affected by both intrinsic light leakage at voltage-off state and residual birefringence after voltage actuation. Table II lists the intrinsic contrast ratio before voltage actuation at $\lambda=514 \mathrm{~nm}$. The contrast ratio for sample II has been greatly improved using circular polarizers. Although sample II cell 1 shows a low contrast ratio because the scattering degrades the dark state, the contrast ratio for cell 2 is comparable to sample I cell 1 . The contrast ratio for $\lambda=633 \mathrm{~nm}$ would be lower because it is closer to the reflection band and the broadband circular polarizer is not designed for reflection band at $\sim 600 \mathrm{~nm}$. After driving, the contrast ratio would also be degraded due to the residual birefringence.

One drawback for the configuration in Fig. 2(a) is that reflection could be observed from the viewer's side under strong ambient light because the BPLC cell reflects lefthanded circular light and it can pass through the LCP. Another possible configuration is to use LCP as the first polarizer and RCP as the second polarizer. The dark state will be similar to the configuration in Fig. 2(a) and there is negligible reflection from viewer's side, but the transmittance will decrease because of the reflection of backlight. However, we can tune the reflection band of BPLC out of the transmission spectrum of RGB color filters or RGB LEDs. Therefore, high transmittance can still be maintained.

In conclusion, we have demonstrated a low voltage blue phase liquid crystal device by red-shifting the pitch length and using circular polarizers. The driving voltage is reduced by $35 \%$ and the Kerr constant is improved by $2.3 \times$. The response time is still in submillisecond range. The contrast ratio is comparable to the BPLC sample with a short pitch length. If we further use a larger Kerr constant material, i.e., Chisso JC-BP01M, ${ }^{13}$ the driving voltage is expected to be reduced from $33 \mathrm{~V}$ to $22 \mathrm{~V}$ with our IPS 5-5 structure at $\lambda=514 \mathrm{~nm}$. With a slightly protruded electrode, the operation voltage could be reduced to below $10 \mathrm{~V}$ for low power consumption.

The authors would like to thank AU Optronics for providing the circular polarizers.

${ }^{1}$ H. Kikuchi, M. Yokota, Y. Hisakado, H. Yang, and T. Kajiyama, Nature Mater. 1, 64 (2002).

${ }^{2}$ Y. Hisakado, H. Kikuchi, T. Nagamura, and T. Kajiyama, Adv. Mater. 17, 96 (2005).

${ }^{3}$ J. Yan, L. Rao, M. Jiao, Y. Li, H. C. Cheng, and S. T. Wu, J. Mater. Chem. 21, 7870 (2011).

${ }^{4}$ K. M. Chen, S. Gauza, H. Xianyu, and S. T. Wu, J. Disp. Technol. 6, 49 (2010).

${ }^{5}$ Y. Chen, J. Yan, J. Sun, S. T. Wu, X. Liang, S. H. Liu, P. J. Hsieh, K. L. Cheng, and J. W. Shiu, Appl. Phys. Lett. 99, 201105 (2011).

${ }^{6} \mathrm{Z}$. Ge, S. Gauza, M. Jiao, H. Xianyu, and S. T. Wu, Appl. Phys. Lett. 94, 101104 (2009).

${ }^{7}$ Z. Ge, L. Rao, S. Gauza, and S. T. Wu, J. Disp. Technol. 5, 250 (2009).

${ }^{8}$ L. Rao, Z. Ge, S. T. Wu, and S. H. Lee, Appl. Phys. Lett. 95, 231101 (2009).

${ }^{9}$ S. Yoon, M. Kim, M. S. Kim, B. G. Kang, M. K. Kim, A. K. Srivastava, S. H. Lee, Z. Ge, L. Rao, S. Gauza, and S. T. Wu, Liq. Cryst. 37, 201 (2010).

${ }^{10}$ M. Jiao, Y. Li, and S. T. Wu, Appl. Phys. Lett. 96, 011102 (2010).

${ }^{11}$ H. C. Cheng, J. Yan, T. Ishinabe, and S. T. Wu, Appl. Phys. Lett. 98, 261102 (2011).

${ }^{12}$ J. Yan and S. T. Wu, Opt. Mater. Express 1, 1527 (2011). 
${ }^{13}$ L. Rao, J. Yan, S. T. Wu, S. Yamamoto, and Y. Haseba, Appl. Phys. Lett. 98, 081109 (2011).

${ }^{14}$ M. Wittek, N. Tanaka, D. Wilkes, M. Bremer, D. Pauluth, M. K. Memmer, J. Canisius, A. Yeh, R. Yan, and K. Skjonnemand, SID Int. Symp. Digest Tech. Papers 43, 25 (2012).

${ }^{15}$ J. Yan and S. T. Wu, J. Disp. Technol. 7, 490 (2011).

${ }^{16}$ T. N. Oo, T. Mizunuma, Y. Nagano, H. Ma, Y. Ogawa, Y. Haseba, H. Higuchi, Y. Okumura, and H. Kikuchi, Opt. Mater. Express 1, 1502 (2011).

${ }^{17}$ C. Y. Fan, H. C. Jau, T. H. Lin, F. C. Yu, T. H. Huang, C. Liu, and N. Sugiura, J. Disp. Technol. 7, 615 (2011).

${ }^{18}$ J. Yan, H. C. Cheng, S. Gauza, Y. Li, M. Jiao, L. Rao, and S. T. Wu, Appl. Phys. Lett. 96, 071105 (2010).

${ }^{19}$ P. R. Gerber, Mol. Cryst. Liq. Cryst. 116, 197 (1985).

${ }^{20}$ H. Kikuchi, Liquid Crystalline Blue Phases (Springer, Berlin,2008), p. 106.

${ }^{21}$ P. Nayek, H. Jeong, H. R. Park, S. W. Kang, S. H. Lee, H. S. Park, H. J. Lee, and H. S. Kim, Appl. Phys. Express 5, 051701 (2012).
${ }^{22}$ H. Y. Liu, C. T. Wang, C. Y. Hsu, and T. H. Lin, Appl. Opt. 50, 1606 (2011).

${ }^{23}$ R. M. Hornreich and S. Shtrikman, Phys. Rev. A 28, 1791 (1983).

${ }^{24}$ C. Bohley and T. Scharf, J. Opt. A, Pure Appl. Opt. 6, S77 (2004).

${ }^{25}$ S. Yokoyama, S. Mashiko, H. Kikuchi, K. Uchida, and T. Nagamura, Adv. Mater. 18, 48 (2006).

${ }^{26}$ F. Castles, F. V. Day, S. M. Morris, D. H. Ko, D. J. Gardiner, M. M. Qasim, S. Nosheen, P. J. W. Hands, S. S. Choi, R. H. Friend, and H. J. Coles, Nature Mater. 11, 599 (2012).

${ }^{27}$ J. Yan, Y. Li, and S. T. Wu, Opt. Lett. 36, 1404 (2011).

${ }^{28}$ K. M. Chen, S. Gauza, H. Xianyu, and S. T. Wu, J. Disp. Technol. 6, 318 (2010).

${ }^{29}$ J. Yan, Y. Chen, S. T. Wu, S. H. Liu, K. L. Cheng, and J. W. Shiu, J. Appl. Phys. 111, 063103 (2012).

${ }^{30}$ L. Rao, J. Yan, S. T. Wu, Y. C. Lai, Y. H. Chiu, H. Y. Chen, C. C. Liang, C. M. Wu, P. J. Hsieh, S. H. Liu, and K. L. Cheng, J. Disp. Technol. 7, 627 (2011). 